Fischer, J.A., Blum, J.W. \& Binswanger, U. (1973) Acute parathyroid hormone response to epinephrine in vivo. Journal of Clinical Investigation, 52, 2434.

Kukreja, S.C., Hargis, G.K., Rosenthal, I.M. \& Williams, G.A. (1973) Pheochromocytoma causing excessive parathyroid hormone production and hypercalcemia. Annals of Internal Medicine, 79, 838.

Miller, S.M., Sizemore, G.W., Sheps, S.G. \& Tyce, G.M. (1975) Parathyroid function in patients with pheochromocytoma. Annals of Internal Medicine, 82, 372.
Riggs, B.L., Arnaud, C.D., Reynolds, J.C. \& Smith, L.H. (1971) Immunologic differentiation of primary hyperparathyroidism from hyperparathyroidism due to non-parathyroid cancer. Journal of Clinical Investigation, 50, 2079.

Swinton, N.W., JR, Clerkin, E.P. \& Flint, L.D. (1972) Hypercalcemia and familial pheochromocytoma: correction after adrenalectomy. Annals of Internal Medicine, 76, 455.

\title{
A case of subacute bacterial endocarditis treated with parenteral clindamycin (clindamycin-2-phosphate)
}

\author{
R. FREeman \\ M.B., Ch.B.
}

\author{
D. W. ROBERTS \\ F.I.M.L.T.
}

\section{Department of Microbiology, University of Leeds}

\section{Summary}

A case of subacute bacterial endocarditis which was due to an $\alpha$-haemolytic streptococcus is described. A therapeutic dilemma arose when the patient developed a drug fever to penicillin and was found to be allergic to cephalosporine. Oral clindamycin was used, but had to be abandoned since severe exacerbation of an unsuspected duodenal ulcer resulted. The patient was finally successfully treated with clindamycin-2-phosphate, a new injectable form of the drug, and the success of this therapy is documented.

\section{Introduction}

Clindamycin-2-phosphate is a new derivative of clindamycin which has been formulated for parenteral use, and which has recently been available for clinical trial in the U.K. We report the following case of subacute bacterial endocarditis due to an $\alpha$-haemolytic streptococcus which was successfully treated with this drug.

\section{Case history}

The patient was a 34-year-old man who had been admitted to another hospital complaining of weight loss, dyspnoea, malaise, anorexia and night sweats. The illness had slowly progressed over the previous month. There was no history of heart disease or of rheumatic fever, but the patient had undergone den-

Correspondence: Dr R. Freeman, Department of Microbiology, University of Leeds, Leeds 2 . tal scaling 2 months previously. Since heart disease was not suspected at that time no prophylactic antibiotics had been given. Admission took place on 26 December 1974.

On examination the patient had gross aortic incompetence, splinter haemorrhages and splenomegaly. He was in moderate left ventricular failure and was clinically anaemic.

Investigation confirmed the presence of anaemia (haemoglobin $9.0 \mathrm{~g} / 100 \mathrm{ml}$ ) and revealed a neutrophil leucocytosis and a raised erythrocyte sedimentation rate (ESR) at $80 \mathrm{~mm} / \mathrm{hr}$. Blood cultures taken on admission yielded a pure growth of an $\alpha$-haemolytic Streptococcus (S. viridans) which was reported as sensitive to penicillin.

The patient was treated with digoxin and frusemide to control the left ventricular failure and was given oral ferrous sulphate for his anaemia. Specific therapy was instituted with benzyl penicillin at a dose of $2 \mathrm{Mu}(1.2 \mathrm{~g})$ given 4-hourly intramuscularly (i.m.).

There was an initial good response, the fever which had been present on admission settling within $24 \mathrm{hr}$ and the patient claiming to feel much better. The left ventricular failure was well controlled.

After 6 days the patient became febrile once more, although he claimed to be feeling much better, and it was thought wise to increase the penicillin to a dose of $4 \mathrm{Mu}(2.4 \mathrm{~g})$ 4-hourly, and to give this intravenously. The fever continued and after a further 
TABLE 1. Clindamycin serum levels during parenteral therapy and results of bactericidal tests

\begin{tabular}{cccccc}
\hline \multirow{2}{*}{ Date } & $\begin{array}{c}\text { Day of } \\
\text { therapy }\end{array}$ & \multicolumn{2}{c}{ Clindamycin level $(\mu \mathrm{g} / \mathrm{ml})$} & \multicolumn{2}{c}{ Bactericidal titre } \\
Peak $(3 \mathrm{hr})$ & Trough $(6 \mathrm{hr})$ & Peak $(3 \mathrm{hr})$ & Trough (6 hr) \\
\hline $4 / 2 / 75$ & 4 th & $5 \cdot 0$ & $3 \cdot 3$ & $1 / 64$ & $1 / 32$ \\
$12 / 2 / 75$ & 12 th & 4.8 & 3.0 & $1 / 32$ & $1 / 32$ \\
\hline
\end{tabular}

TABLE 2. Results of haematological and biochemical tests performed before, during and after parenteral clindamycin therapy

\begin{tabular}{|c|c|c|c|c|c|}
\hline \multirow[t]{2}{*}{ Test } & \multirow[t]{2}{*}{ Before } & \multicolumn{2}{|c|}{ During clindamycin therapy } & \multirow[t]{2}{*}{ After } & \multirow[t]{2}{*}{ Normal range } \\
\hline & & Early & Late & & \\
\hline Haemoglobin & $9 \cdot 0$ & $9 \cdot 8$ & $10 \cdot 2$ & $14 \cdot 0$ & $13 \cdot 5-18.0 \mathrm{~g} / 100 \mathrm{ml}$ \\
\hline WCC & $13 \cdot 0$ & $10 \cdot 0$ & $8 \cdot 5$ & $8 \cdot 6$ & $4.0-11.0 \times 10^{9} / 1$ \\
\hline Neutrophils & 78 & 70 & 83 & 61 & $40-75 \%$ of total \\
\hline Lymphocytes & 18 & 20 & 8 & 25 & $20-40 \%$ of total \\
\hline Monocytes & 4 & 10 & 7 & 10 & $2-10 \%$ of total \\
\hline Eosinophils & 1 & 1 & 2 & 4 & $1-6 \%$ of total \\
\hline Platelets & 'Normal' & 'Normal' & 'Normal' & 'Normal' & \\
\hline BUN & $7 \cdot 3$ & $7 \cdot 3$ & $8 \cdot 2$ & $7 \cdot 0$ & $2 \cdot 5-7 \cdot 1 \mathrm{mmol} / 1$ \\
\hline SGOT & 22 & 24 & 19 & 18 & 4-40 i.u. \\
\hline SGPT & 24 & 21 & 20 & 16 & less than 22 i.u. \\
\hline Alk.P. & 7 & 8 & 6 & 7 & $3-13 u$ \\
\hline Bilirubin & 4 & 14 & 4 & 2 & 0-15 mmol/1 \\
\hline \\
\hline WBC & Absent & Absent & Absent & Absent & up to $10 \times 10^{6} / 1$ \\
\hline $\mathbf{R B C}$ & $10 \times 10^{6}$ & Nil & Nil & Nil & up to $3 \times 10^{6} / 1$ \\
\hline Casts & Nil & Nil & Nil & Nil & - \\
\hline
\end{tabular}

WCC, white cell count; BUN, blood urea nitrogen; SGOT, serum glutamate oxaloacetate transaminase; SGPT, serum glutamate pyruvate transaminase; Alk.P., alkaline phosphatase; RBC, red blood cells; WBC, white blood cells.

week gentamicin at a dose of $80 \mathrm{mg}$ 8-hourly i.m. was added to the penicillin.

This combination of drugs failed to produce defervescence, and on 21 January 1975 the patient was transferred to the Leeds General Infirmary for further treatment.

On transfer although the patient remained febrile he claimed to be feeling quite well, and it was thought, since the isolated organism was of a type which was commonly very sensitive to penicillin, that his fever, which had appeared at the beginning of the second week of penicillin therapy, might be a drug fever (Dormer, 1966). This view was further supported when the isolated organism was re-examined and confirmed to be a typical $\alpha$-haemolytic streptococcus, the minimum inhibitory concentration (MIC) of which to penicillin was 0.03 $\mu \mathrm{g}$. Accordingly, on transfer all antibiotics were stopped and $24 \mathrm{hr}$ later therapy was re-instituted with cephradine, $1.0 \mathrm{~g}$ 6-hourly i.m. Unfortunately, the patient developed an urticarial eruption after only six doses and this drug was then withdrawn on the basis of presumed cephalosporin allergy.

In an attempt to employ a drug with good bactericidal activity against $\alpha$-haemolytic streptococci and which was totally unrelated to the penicillin family the sensitivity of the organism to clindamycin was examined. The MIC of the organism to clindamycin (using pure clindamycin powder to make the testing solutions) was found to be less than $0.015 \mu \mathrm{g}$. The patient was therefore given oral clindamycin at a dose of $300 \mathrm{mg}$ 6-hourly. There was an excellent response to this therapy and his fever immediately settled. However, over the next 8 days he experienced increasing dyspepsia and this was exacerbated by the oral clindamycin. A barium meal examination revealed a large duodenal ulcer, and the changes were thought to represent a well established ulcer which, although exacerbated by the drug, was unlikely to have been caused by it.

Because of the problems anticipated in reverting to drugs of the penicillin family it was decided to continue clindamycin therapy with parenteral clindamycin. The patient was given clindamycin2-phosphate $300 \mathrm{mg}$ i.m. 6-hourly, and this was continued for a further 20 days, to complete a total course of 28 days (oral + parenteral), this length of treatment being thought to be adequate for the eradication of the infection (Weinstein and Schlesinger, 1973) During this therapy the opportunity was taken to measure the serum levels of clindamycin achieved. Blood was taken $3 \mathrm{hr}$ after an injection and 
just before an injection, the samples reflecting peak and trough concentrations respectively. Serum was separated immediately after collection and an equal volume of phosphate buffer ( $\mathrm{pH} \mathrm{8.0)}$ was added to prevent hydrolysis of any microbiologically inactive clindamycin-2-phosphate to ensure that only free clindamycin was assayed. The results of these assays which were done on two occasions during therapy are found in Table 1. More importantly, the ability of the patient's serum to kill the isolated organism was also assessed, it being widely accepted that if the patient's serum will kill the organism at a dilution of $1 / 4$, or greater, then optimal therapy of the infection is in progress. These results are also found in Table 1.

The patient completed his course of therapy with no further incidents, and all antibiotics were then discontinued. By this time he was well, he was no longer anaemic (haemoglobin $14.0 \mathrm{~g} / 100 \mathrm{ml}$ ) and was afebrile. The ESR had fallen to $30 \mathrm{~mm} / \mathrm{hr}$.

Although his left ventricular failure was controlled by digoxin and frusemide, cardiac catheterization studies indicated that the aortic valve was severely incompetent, and he proceeded to aortic valve replacement on 25 February 1975. Histology of the removed valve showed the typical changes of infective endocarditis, but no evidence of activity was found, indeed healing was well established. Similarly, culture of the excised valve failed to isolate any organism. The patient was not given any antibiotic treatment post-operatively and had made an uneventful recovery from his operation. When last seen in the out-patient clinic 4 months after surgery he was well and there were no signs of infection.

Since clindamycin-2-phosphate was a new drug undergoing clinical trial at the time of this case, various haematological and biochemical parameters were measured before, during and after exhibition of the drug. These are summarized in Table 2.

\section{Discussion}

The complicated course of this patient's illness illustrates some of the problems encountered in the treatment of infective endocarditis, and also allows a preliminary evaluation of the efficacy of clindamycin in the treatment of endocarditis due to $\alpha$-haemolytic streptococci.

Thus, in the assessment of the effectiveness of therapy it can be seen that such simple clinical parameters as temperature can be very misleading, and this case illustrates the value of the so-called 'bactericidal test' (Garrod, Lambert and O'Grady,
1973). Using this simple laboratory procedure it can be seen from the data in Table 1 that the patient's serum killed the isolated organism at dilutions well in excess of $1 / 4$, thus indicating that treatment was effective. There is little doubt, taking into consideration the low MIC of the organism to penicillin, that the application of this test at the time when the patient's fever first recurred in the first hospital would have resolved the therapeutic dilemma, and would have shown in all probability that effective treatment was being given, and that by inference the fever was not due to uncontrolled infection. Similarly, when this test was applied during the clindamycin therapy it indicated effective treatment and this was borne out by subsequent events.

Turning to the use of parenteral clindamycin, Table 1 shows that adequate serum levels were achieved on the dose schedule given, and Table 2 reveals that this was at no haematological or biochemical cost to the patient. The sole abnormalities before treatment was commenced were albuminuria and haematuria, both of which are to be expected in infective endocarditis and both of which resolved during the treatment course.

It is obviously premature to suggest that clindamycin in either oral or parenteral form should be adopted as a common treatment of infective endocarditis due to $\alpha$-haemolytic streptococci, but we feel that it should be considered as being of potential use in this condition, especially in the patient in whom penicillin is contra-indicated. We would also urge microbiologists to include sensitivity testing to this drug in the examination of $\alpha$-haemolytic streptococci isolated from blood cultures.

\section{Acknowledgments}

We thank Mr M. I. Ionescu and Dr D. R. Smith, under whose joint care this patient remains, for their help and interest. We are also grateful to the Upjohn Company for clindamycin powder, advice on assays of clindamycin in the presence of clindamycin-2-phosphate and for the provision of parenteral clindamycin.

\section{References}

DORMER, A.E. (1966) Septicaemia and endocarditis. In: The Therapeutic Use of Antibiotics in Hospital Practice (Ed. by M. Ridley and I. Phillips), pp. 181-190. Livingstone, Edinburgh and London.

Garrod, L.P., LAMBert, H.P. \& O'Grady. F. (1973) In: Antibiotics and Chemotherapy, p. 529. Livingstone, Edinburgh and London.

Weinstein, L. \& Schlesinger, J. (1973) Treatment of infective endocarditis. Progress in Cardiovascular Diseases, 16, 275. 